\title{
Exact dipole radiation for an oblate spheroidal cavity filled with isorefractive material and aperture-coupled to a half space
}

\author{
Cristian Berardi, Student Member, IEEE, Danilo Erricolo, Senior Member, IEEE, \\ Piergiorgio L. E. Uslenghi, Fellow, IEEE
}

\begin{abstract}
An oblate semi-spheroidal cavity flush-mounted under a metal plane and coupled to the half-space above it via its circular interfocal aperture is considered. The cavity is filled with a material isorefractive to the medium that occupies the halfspace above it. An exact solution is obtained for the radiation of an electric or magnetic dipole located on the symmetry axis of the structure, either outside or inside the cavity, and axially oriented. Numerical results are provided.
\end{abstract}

Index Terms-Electromagnetic radiation, complex media, spheroidal functions, isorefractive media, exact solution, series expansion, mode coupling

\section{INTRODUCTION}

A $\mathrm{N}$ oblate semi-spheroidal cavity with metallic walls is flush-mounted under an infinite metal plane, and is coupled to the half-space above the plane via an aperture equal to the interfocal circle of the spheroidal coordinate system. The cavity is filled with a material that is isorefractive to the medium occupying the half-space above the metal plane. The primary source is an electric or magnetic dipole located on the axis of symmetry of the structure and axially oriented. The electromagnetic fields are represented everywhere by infinite series of oblate spheroidal wave functions, whose unknown expansion coefficients are determined analytically by imposing the boundary conditions, as was done in [1] for the radiation from a metallic spheroid. The notation for the spheroidal wave functions is that of Flammer [2].

Preliminary results of this research were given in [3]. In the literature, there are at least two other works that provide analytical results for radiation from spheroidal structures. Alexopoulos et al. [4] investigated a spheroidal object with an impedance surface for axial dipole excitation. Sebak and Sinha [5] considered a conducting spheroidal object coated with a dielectric for an axially incident plane wave.

The exact solutions obtained herein are important for at least two reasons. First, they constitute new exact solutions for boundary-value problems, thus enriching the catalog of available canonical solutions. Second, they constitute a good validation test for frequency-domain computer codes, because they contain challenging features such as two different penetrable materials, sharp edges, and a cavity. The technique used in this paper is similar to the one employed to solve exactly

The authors are with the Department of Electrical and Computer Engineering, University of Illinois at Chicago, Chicago, IL 60607 USA. Email: derricol@ece.uic.edu, uslenghi@uic.edu. This work was supported by the U.S. Department of Defense under MURI grant F49620-01-1-0436. the two-dimensional problem of radiation and scattering for a slotted semi-elliptical channel under a metal plane [6]. However, it should be pointed out that axial symmetry of both the structure and the primary field is required for exact analytical solutions of boundary value problems in spheroidal coordinates to be obtainable by separation of variables and imposition of the boundary conditions.

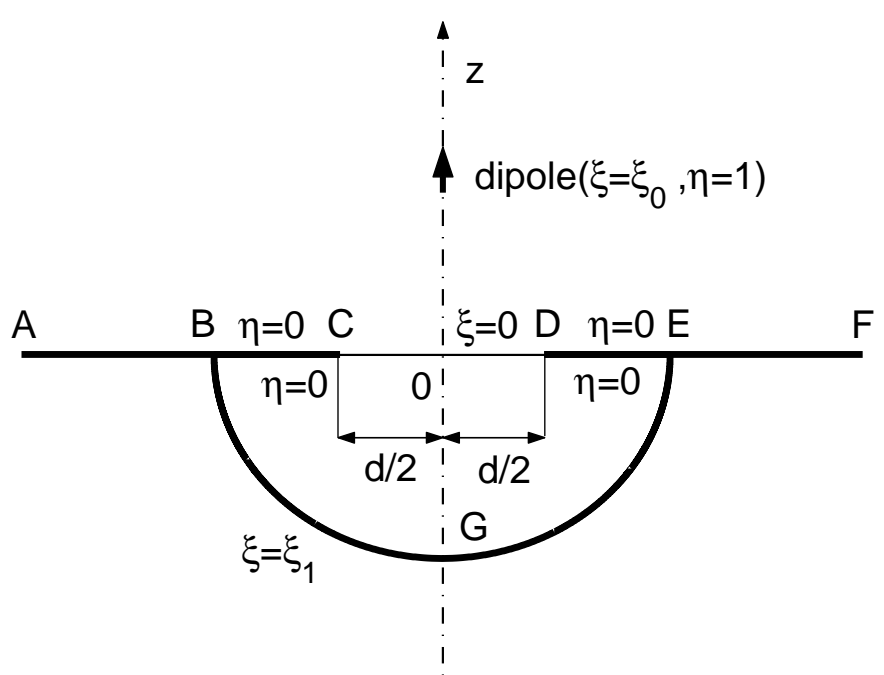

Fig. 1. Geometry of the problem.

The geometry of the problem is presented in Section II. Exact solutions for the radiation by electric and magnetic dipole sources are obtained for dipoles either outside the cavity (section III) or inside it (section IV). Numerical results are presented and discussed in section V. Finally, some useful properties of the oblate spheroidal functions are collected in the Appendix. The time-dependence factor $\exp (-i \omega t)$ is omitted throughout.

\section{Geometry of the Problem}

A cross section of the structure through its axis $z$ of symmetry is shown in Fig. 1. The cut $B G E$ of the oblate semi-spheroidal cavity is a semi-ellipse with major axis $B E$ and interfocal distance $C D=d$. The aperture connecting the cavity to the half-space $z>0$ above the metallic plane $A F$ is a circular hole with center $O$ at the origin of coordinates and diameter $d$. The oblate spheroidal coordinates $(\eta, \xi, \varphi)$ are 
a right-handed system related to the rectangular coordinates $(x, y, z)$ by:

$$
\left\{\begin{array}{l}
x=\frac{d}{2} \sqrt{\left(\xi^{2}+1\right)\left(1-\eta^{2}\right)} \cos \varphi \\
y=\frac{d}{2} \sqrt{\left(\xi^{2}+1\right)\left(1-\eta^{2}\right)} \sin \varphi \\
z=\frac{d}{2} \xi \eta
\end{array}\right.
$$

where $0 \leq \xi<\infty,-1 \leq \eta \leq 1,0 \leq \varphi \leq 2 \pi$. The inverse transformation from rectangular coordinates to oblate spheroidal coordinates is reported here for convenience:

$$
\left\{\begin{array}{l}
\varphi=\arctan \frac{y}{x} \\
\eta=\frac{z}{|z|} \sqrt{\frac{-\left[4\left(x^{2}+y^{2}+z^{2}\right)-d^{2}\right]+\sqrt{4\left[\left(x^{2}+y^{2}+z^{2}\right)-d^{2}\right]^{2}+16 d^{2} z^{2}}}{2 d^{2}}} \\
\xi=\sqrt{\frac{\left[4\left(x^{2}+y^{2}+z^{2}\right)-d^{2}\right]+\sqrt{4\left[\left(x^{2}+y^{2}+z^{2}\right)-d^{2}\right]^{2}+16 d^{2} z^{2}}}{2 d^{2}}}
\end{array}\right.
$$

The surface $\xi=$ constant is an oblate spheroid (i.e. a flattened ellipsoid of revolution) with major axis $d \sqrt{\xi^{2}+1}$ and minor axis $d \xi$, with $\mathrm{z}$ as the symmetry axis; the circular aperture of diameter $d$ that lies in the $(x, y)$ plane and is centered at the origin corresponds to $\xi=0$, whereas the curved wall of the cavity corresponds to half the spheroid $\xi=\xi_{1}$. The surface $|\eta|=$ constant is a hyperboloid of revolution with $z$ as the symmetry axis, $\eta>0(\eta<0)$ for $z>0(z<0)$, and asymptotic cone of semi-aperture $\theta=\arccos \eta$; in particular, $|\eta|=1$ is the $\mathrm{z}$-axis, whereas $\eta=0$ is the $z=0$ plane with the circle $\xi=0$ excluded. The surface $\varphi=$ constant is a half-plane originating in the z-axis. The medium occupying the half-space $z>0$ is characterized by an electric permittivity $\varepsilon$ and a magnetic permeability $\mu$, whereas the cavity is filled with a material of permittivity $\varepsilon_{1}$ and permeability $\mu_{1}$. The two media are isorefractive, hence they have the same propagation constant

$$
k=\omega \sqrt{\varepsilon \mu}=\omega \sqrt{\varepsilon_{1} \mu_{1}},
$$

but, in general, different intrinsic impedances:

$$
Z=Y^{-1}=\sqrt{\frac{\mu}{\varepsilon}} \neq Z_{1}=Y_{1}^{-1}=\sqrt{\frac{\mu_{1}}{\varepsilon_{1}}} .
$$

The primary source is an electric or magnetic dipole located on the $\mathrm{z}$-axis and axially oriented. The case of a dipole at $\left(\xi=\xi_{0}, \eta=1\right)$ on the positive $\mathrm{z}$-axis (see Fig. 1) is discussed in section III, whereas the case of a dipole at $\left(\xi=\xi_{0}, \eta=-1\right)$ inside the cavity is examined in section IV. It should be noted that the electric and magnetic dipole cases are not the dual of each other, because the metal boundaries are considered perfect electric conductors on both cases.

\section{DIPOLE SOURCES OUTSIDE THE CAVITY}

\section{A. Electric dipole}

For a primary electric source located on the $\mathrm{z}$-axis and axially oriented, the electric and magnetic fields everywhere are of the type:

$$
\begin{array}{ll}
\underline{E}=E_{\xi}(\xi, \eta) \widehat{\xi}+E_{\eta}(\xi, \eta) \widehat{\eta}, & E_{\varphi}=0, \\
\underline{H}=H_{\varphi}(\xi, \eta) \widehat{\varphi}, & H_{\xi}=H_{\eta}=0,
\end{array}
$$

where

$$
\begin{aligned}
& E_{\xi}=-\frac{i Z}{c} \sqrt{\frac{1-\eta^{2}}{\xi^{2}+\eta^{2}}}\left(\frac{\partial}{\partial \eta}-\frac{\eta}{1-\eta^{2}}\right) H_{\varphi} \\
& E_{\eta}=\frac{i Z}{c} \sqrt{\frac{\xi^{2}+1}{\xi^{2}+\eta^{2}}}\left(\frac{\partial}{\partial \xi}+\frac{\xi}{\xi^{2}+1}\right) H_{\varphi}
\end{aligned}
$$

and $c=k d / 2$ is the product of the wavenumber and the interfocal radius. For fields inside the cavity, we add the subscript 1 to the field components and replace $Z$ with $Z_{1}$.

An electric dipole located at $\left(\xi_{0}, \eta_{0}=1\right)$ on the positive $\mathrm{z}$ axis and axially oriented, with moment $\widehat{z} 4 \pi \varepsilon / k$ corresponding to an electric Hertz vector $\underline{\Pi}^{(e)}=\widehat{z} \exp (i k R) /(k R)$ where $R$ is the distance of the observation point $(\eta, \xi, \phi)$ from the dipole, generates a primary magnetic field [1]:

$$
\begin{aligned}
H_{\varphi}^{i}= & \frac{2 k^{2} Y}{\sqrt{\xi_{0}^{2}+1}} \sum_{n=1}^{\infty} \frac{(-i)^{n}}{\widetilde{\rho}_{1, n} \widetilde{N}_{1, n}} \times \\
& R_{1, n}^{(1)}\left(-i c, i \xi_{<}\right) R_{1, n}^{(3)}\left(-i c, i \xi_{>}\right) S_{1, n}(-i c, \eta),
\end{aligned}
$$

where $R_{1, n}^{(1),(3)}$ are radial oblate spheroidal functions of the first and third kind, respectively, and $S_{1, n}$ are angular oblate spheroidal functions [2]. The quantity $\xi_{<}\left(\xi_{>}\right)$is the smaller (larger) between $\xi$ and $\xi_{0}$. It should be noted that the summation in (8) extends to all $n \geq 1$, not $n \geq 0$ as erroneously stated in [1]. The total field in the absence of the cavity is

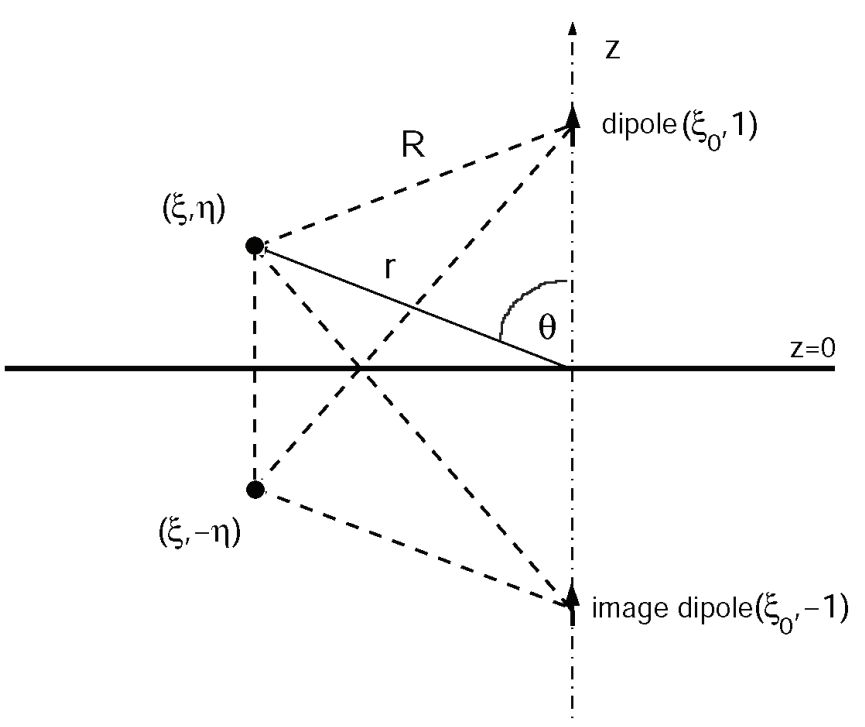

Fig. 2. Incident and reflected fields for electric dipole source

the field of the dipole above the PEC plane $z=0$, that is the superposition of the primary field and the reflected field due to the image dipole located at $\left(\xi_{0},-1\right)$. The field $H_{\varphi}^{r}$ produced at $(\xi, \eta)$ by the image dipole equals the field $H_{\varphi}^{i}$ produced at $(\xi,-\eta)$ by the original dipole (see Fig. 2):

$$
H_{\varphi}^{r}(\xi, \eta)=H_{\varphi}^{i}(\xi,-\eta)
$$


hence, the total field at $(\xi, \eta)$ is:

$$
\begin{array}{r}
H_{\varphi}^{i}+H_{\varphi}^{r}=\frac{2 k^{2} Y}{\sqrt{\xi_{0}^{2}+1}} \sum_{n=1}^{\infty} \frac{(-i)^{n}}{\widetilde{\rho}_{1, n} \widetilde{N}_{1, n}} R_{1, n}^{(1)}\left(-i c, i \xi_{<}\right) \times \\
R_{1, n}^{(3)}\left(-i c, i \xi_{>}\right)\left[S_{1, n}(-i c, \eta)+S_{1, n}(-i c,-\eta)\right],
\end{array}
$$

which, with the aid of (66) and (67) in appendix, may be rewritten as:

$$
\begin{aligned}
& H_{\varphi}^{i}+H_{\varphi}^{r}=-\frac{4 i k^{2} Y}{\sqrt{\xi_{0}^{2}+1}} \sum_{l=0}^{\infty} \frac{(-1)^{l}}{\widetilde{\rho}_{1,2 l+1} \widetilde{N}_{1,2 l+1}} \times \\
& R_{1,2 l+1}^{(1)}\left(-i c, i \xi_{<}\right) R_{1,2 l+1}^{(3)}\left(-i c, i \xi_{>}\right) S_{1,2 l+1}(-i c, \eta) .
\end{aligned}
$$

In the presence of the cavity, the total magnetic field is:

$$
\begin{aligned}
H_{\varphi} & =H_{\varphi}^{i}+H_{\varphi}^{r}+H_{\varphi}^{d} & & \text { for } z \geq 0 \\
& =H_{1 \varphi} & & \text { for } z \leq 0
\end{aligned}
$$

where

$$
\begin{aligned}
H_{\varphi}^{d}=-\frac{4 i k^{2} Y}{\sqrt{\xi_{0}^{2}+1}} \sum_{l=0}^{\infty} \frac{(-1)^{l} a_{l}^{(e)}}{\widetilde{\rho}_{1,2 l+1} \widetilde{N}_{1,2 l+1}} \times \\
R_{1,2 l+1}^{(3)}(-i c, i \xi) S_{1,2 l+1}(-i c, \eta)
\end{aligned}
$$

is the diffracted field due to the cavity and the coupling hole, that satisfies the radiation condition at infinity as well as the boundary condition on the metal plane $z=0$ outside the hole, while

$$
\begin{array}{r}
H_{1 \varphi}=-\frac{4 i k^{2} Y_{1}}{\sqrt{\xi_{0}^{2}+1}} \sum_{l=0}^{\infty} \frac{(-1)^{l} b_{l}^{(e)}}{\widetilde{\rho}_{1,2 l+1} \widetilde{N}_{1,2 l+1}}\left[R_{1,2 l+1}^{(1)}(-i c, i \xi)+\right. \\
\left.c_{l}^{(e)} R_{1,2 l+1}^{(3)}(-i c, i \xi)\right] S_{1,2 l+1}(-i c, \eta)
\end{array}
$$

is the total field inside the cavity, and satisfies the boundary condition on the flat annular ring $\left(0 \leq \xi \leq \xi_{1}, \eta=0\right)$. The modal coefficients $a_{l}^{(e)}$ in (13), and $b_{l}^{(e)}$ and $c_{l}^{(e)}$ in (14), are determined by imposing the boundary condition

$$
\left.E_{1 \eta}\right|_{\xi=\xi_{1}}=0
$$

on the curved portion of the cavity's surface, and the continuity of the tangential components of the total electric and magnetic fields across the aperture $\xi=0$ :

$$
\begin{aligned}
\left(H_{\varphi}^{i}+H_{\varphi}^{r}+H_{\varphi}^{d}\right)_{\xi=0, \eta} & =\left.H_{1 \varphi}\right|_{\xi=0,-\eta}, \\
\left.E_{\eta}^{d}\right|_{\xi=0, \eta} & =-\left.E_{1 \eta}\right|_{\xi=0,-\eta},
\end{aligned}
$$

where it is noted that the unit vector $\hat{\eta}$ is in opposite directions at $z= \pm 0$, that $\eta$ changes sign abruptly when the $z=0$ plane is crossed, and that $E_{\eta}^{i}+E_{\eta}^{r}=0$ at $\xi=0$. Condition (15), with the aid of (7) yields:

$$
c_{l}^{(e)}=-\frac{R_{1,2 l+1}^{(1)^{\prime}}\left(-i c, i \xi_{1}\right)+\frac{\xi_{1}}{\xi_{1}^{2}+1} R_{1,2 l+1}^{(1)}\left(-i c, i \xi_{1}\right)}{R_{1,2 l+1}^{(3)^{\prime}}\left(-i c, i \xi_{1}\right)+\frac{\xi_{1}}{\xi_{1}^{2}+1} R_{1,2 l+1}^{(3)}\left(-i c, i \xi_{1}\right)}
$$

where the prime means derivative with respect to $\xi$, evaluated at $\xi=\xi_{1}$. Conditions (16) and (17) yield:

$$
\begin{aligned}
& a_{l}^{(e)}=-c_{l}^{(e)} b_{l}^{(e)}= \\
& -\frac{c_{l}^{(e)} R_{1,2 l+1}^{(1)}(-i c, i 0) R_{1,2 l+1}^{(3)}\left(-i c, i \xi_{0}\right)}{\zeta R_{1,2 l+1}^{(1)}(-i c, i 0)+(1+\zeta) c_{l}^{(e)} R_{1,2 l+1}^{(3)}(-i c, i 0)}
\end{aligned}
$$

where

$$
\zeta=\frac{Z}{Z_{1}} .
$$

The surface current densities on the PEC surfaces are the total tangential magnetic field rotated by $90^{\circ}$. By using (65):

$$
\begin{gathered}
\left.H_{1 \varphi}\right|_{\xi=\xi_{1}}=\frac{4 k^{2} Y_{1}}{c \sqrt{\xi_{0}^{2}+1}} \sum_{l=0}^{\infty} \frac{(-1)^{l} b_{l}^{(e)}}{\widetilde{\rho}_{1,2 l+1} \widetilde{N}_{1,2 l+1}} \times \\
\frac{S_{1,2 l+1}(-i c, \eta)}{\xi_{1} R_{1,2 l+1}^{(3)}\left(-i c, i \xi_{1}\right)+\left(\xi_{1}^{2}+1\right) R_{1,2 l+1}^{(3)^{\prime}}\left(-i c, i \xi_{1}\right)}
\end{gathered}
$$

whereas, by using (69):

$$
\begin{aligned}
\left.H_{1 \varphi}\right|_{\eta=0}= & -\frac{4 i k^{2} Y_{1}}{\sqrt{\xi_{0}^{2}+1}} \sum_{l=0}^{\infty} \frac{(-1)^{l} b_{l}^{(e)} P_{2 l+1}^{1}(0)}{\widetilde{\rho}_{1,2 l+1} \widetilde{N}_{1,2 l+1}} \times \\
& {\left[R_{1,2 l+1}^{(1)}(-i c, i \xi)+c_{l}^{(e)} R_{1,2 l+1}^{(3)}(-i c, i \xi)\right] . }
\end{aligned}
$$

On the surface of the ground plane:

$$
\begin{gathered}
\left(H_{\varphi}^{i}+H_{\varphi}^{r}+H_{\varphi}^{d}\right)_{\eta=0}=-\frac{4 i k^{2} Y}{\sqrt{\xi_{0}^{2}+1}} \sum_{l=0}^{\infty} \frac{(-1)^{l} P_{2 l+1}^{1}(0)}{\widetilde{\rho}_{1,2 l+1} \widetilde{N}_{1,2 l+1}} \times \\
{\left[R_{1,2 l+1}^{(1)}\left(-i c, i \xi_{<}\right) R_{1,2 l+1}^{(3)}\left(-i c, i \xi_{>}\right)+a_{l}^{(e)} R_{1,2 l+1}^{(3)}(-i c, i \xi)\right] .}
\end{gathered}
$$

The diffracted far field is obtained with the aid of the asymptotic expansions in appendix, and by noting that $c \xi \sim k r$ and $\eta \sim \cos \theta$ as $c \xi \rightarrow \infty$ :

$$
\left.H_{\varphi}^{d}\right|_{c \xi \rightarrow \infty} \sim \frac{e^{i k r}}{k r} \frac{4 i k^{2} Y}{\sqrt{\xi_{0}^{2}+1}} \sum_{l=0}^{\infty} \frac{a_{l}^{(e)} S_{1,2 l+1}(-i c, \cos \theta)}{\widetilde{\rho}_{1,2 l+1} \widetilde{N}_{1,2 l+1}}
$$

The limit when the cavity recedes to infinity $\left(c \xi_{1} \rightarrow \infty\right)$, i.e., the case of a circular hole in an infinite metallic screen separating two isorefractive half-spaces, may be obtained from the previous formulas by assuming that $c$ has a vanishingly small positive imaginary part. By using (74-77), we obtain from (18) and (19):

$\left.c_{l}^{(e)}\right|_{c \xi_{1} \rightarrow \infty} \sim-\frac{1}{2} e^{-i 2 c \xi_{1}}$,

$\left.b_{l}^{(e)}\right|_{c \xi_{1} \rightarrow \infty} \sim-\frac{2}{1+\zeta} \frac{R_{1,2 l+1}^{(1)}(-i c, i 0)}{R_{1,2 l+1}^{(3)}(-i c, i 0)} R_{1,2 l+1}^{(3)}\left(-i c, i \xi_{0}\right) e^{i 2 c \xi_{1}}$,

$\left.a_{l}^{(e)}\right|_{c \xi_{1} \rightarrow \infty} \sim-\frac{1}{1+\zeta} \frac{R_{1,2 l+1}^{(1)}(-i c, i 0)}{R_{1,2 l+1}^{(3)}(-i c, i 0)} R_{1,2 l+1}^{(3)}\left(-i c, i \xi_{0}\right)$,

hence, from (13) and (14):

$$
\begin{aligned}
\left.H_{\varphi}^{d}\right|_{c \xi_{1} \rightarrow \infty} & \sim \frac{4 i k^{2} Y}{\sqrt{\xi_{0}^{2}+1}} \frac{1}{1+\zeta} \Sigma^{(e)}, \\
\left.H_{1 \varphi}\right|_{c \xi_{1} \rightarrow \infty} & \sim-\frac{4 i k^{2} Y_{1}}{\sqrt{\xi_{0}^{2}+1}} \frac{1}{1+\zeta} \Sigma^{(e)},
\end{aligned}
$$


where

$$
\begin{aligned}
& \Sigma^{(e)}=\sum_{l=0}^{\infty} \frac{(-1)^{l}}{\widetilde{\rho}_{1,2 l+1} \widetilde{N}_{1,2 l+1}} \frac{R_{1,2 l+1}^{(1)}(-i c, i 0)}{R_{1,2 l+1}^{(3)}(-i c, i 0)} \times \\
& R_{1,2 l+1}^{(3)}\left(-i c, i \xi_{0}\right) R_{1,2 l+1}^{(3)}(-i c, i \xi) S_{1,2 l+1}(-i c, \eta) .
\end{aligned}
$$

\section{B. Magnetic dipole}

The derivations are similar to those for the electric dipole, hence only the results are given. For a primary magnetic source located on the $\mathrm{z}$-axis and axially oriented, the electric and magnetic field everywhere are of the type:

$$
\begin{array}{ll}
\underline{E}=E_{\varphi}(\xi, \eta) \widehat{\varphi}, & E_{\xi}=E_{\eta}=0, \\
\underline{H}=H_{\xi}(\xi, \eta) \widehat{\xi}+H_{\eta}(\xi, \eta) \widehat{\eta}, & H_{\varphi}=0,
\end{array}
$$

where

$$
\begin{aligned}
& H_{\xi}=\frac{i Y}{c} \sqrt{\frac{1-\eta^{2}}{\xi^{2}+\eta^{2}}}\left(\frac{\partial}{\partial \eta}-\frac{\eta}{1-\eta^{2}}\right) E_{\varphi} \\
& H_{\eta}=-\frac{i Y}{c} \sqrt{\frac{\xi^{2}+1}{\xi^{2}+\eta^{2}}}\left(\frac{\partial}{\partial \xi}+\frac{\xi}{\xi^{2}+1}\right) E_{\varphi}
\end{aligned}
$$

for fields inside the cavity, we add the subscript 1 to the field components and replace $Y$ with $Y_{1}$. A magnetic dipole located at $\left(\xi_{0}, \eta_{0}=1\right)$ on the positive $\mathrm{z}$-axis and axially oriented, with moment $\widehat{z} 4 \pi / k$ corresponding to a magnetic Hertz vector $\underline{\Pi}^{(m)}=\widehat{z} \exp (i k R) /(k R)$, generates a primary electric field [1]:

$$
\begin{aligned}
E_{\varphi}^{i}=- & \frac{2 k^{2} Z}{\sqrt{\xi_{0}^{2}+1}} \sum_{n=1}^{\infty} \frac{(-i)^{n}}{\widetilde{\rho}_{1, n} \widetilde{N}_{1, n}} \times \\
& R_{1, n}^{(1)}\left(-i c, i \xi_{<}\right) R_{1, n}^{(3)}\left(-i c, i \xi_{>}\right) S_{1, n}(-i c, \eta) .
\end{aligned}
$$

In the absence of a cavity, the image magnetic dipole into the PEC plane $\mathrm{z}=0$ produces a field $E_{\varphi}^{r}$ such that

$$
E_{\varphi}^{r}(\xi, \eta)=-E_{\varphi}^{i}(\xi,-\eta),
$$

wherefrom, with the aid of (66) and (67):

$$
\begin{gathered}
E_{\varphi}^{i}+E_{\varphi}^{r}=-\frac{4 k^{2} Z}{\sqrt{\xi_{0}^{2}+1}} \sum_{l=1}^{\infty} \frac{(-1)^{l}}{\widetilde{\rho}_{1,2 l} \widetilde{N}_{1,2 l}} R_{1,2 l}^{(1)}\left(-i c, i \xi_{<}\right) \times \\
R_{1,2 l}^{(3)}\left(-i c, i \xi_{>}\right) S_{1,2 l}(-i c, \eta) .
\end{gathered}
$$

The total electric field in the presence of the cavity filled with isorefractive material is:

$$
\begin{aligned}
E_{\varphi} & =E_{\varphi}^{i}+E_{\varphi}^{r}+E_{\varphi}^{d}, & & \text { for } z \geq 0 \\
& =E_{1 \varphi}, & & \text { for } z \leq 0,
\end{aligned}
$$

where

$$
\begin{gathered}
E_{\varphi}^{d}=-\frac{4 k^{2} Z}{\sqrt{\xi_{0}^{2}+1}} \sum_{l=1}^{\infty} \frac{(-1)^{l} a_{l}^{(m)}}{\widetilde{\rho}_{1,2 l} \widetilde{N}_{1,2 l}} R_{1,2 l}^{(3)}(-i c, i \xi) S_{1,2 l}(-i c, \eta), \\
E_{1 \varphi}=-\frac{4 k^{2} Z_{1}}{\sqrt{\xi_{0}^{2}+1}} \sum_{l=1}^{\infty} \frac{(-1)^{l} b_{l}^{(m)}}{\widetilde{\rho}_{1,2 l} \widetilde{N}_{1,2 l}}\left[R_{1,2 l}^{(1)}(-i c, i \xi)+\right. \\
\left.c_{l}^{(m)} R_{1,2 l}^{(3)}(-i c, i \xi)\right] S_{1,2 l}(-i c, \eta),
\end{gathered}
$$

and the modal coefficients are found by imposing the boundary conditions:

$$
\begin{aligned}
& c_{l}^{(m)}=-\frac{R_{1,2 l}^{(1)}\left(-i c, i \xi_{1}\right)}{R_{1,2 l}^{(3)}\left(-i c, i \xi_{1}\right)}, \\
& a_{l}^{(m)}=-\zeta^{-1} b_{l}^{(m)} c_{l}^{(m)}= \\
& \frac{-c_{l}^{(m)} R_{1,2 l}^{()^{\prime}}(-i c, i 0) R_{1,2 l}^{(3)}\left(-i c, i \xi_{0}\right)}{\zeta R_{1,2 l}^{(1)^{\prime}}(-i c, i 0)+(1+\zeta) c_{l}^{(m)} R_{1,2 l}^{(3)^{\prime}}(-i c, i 0)} .
\end{aligned}
$$

The tangential magnetic field on the PEC surfaces is given by:

$$
\begin{gathered}
\left(H_{\xi}^{i}+H_{\xi}^{r}+H_{\xi}^{d}\right)_{\eta=0}=-\frac{4 i k^{2}}{c \xi \sqrt{\xi_{0}^{2}+1}} \sum_{l=1}^{\infty} \frac{(-1)^{l} P_{2 l}^{1^{\prime}}(0)}{\widetilde{\rho}_{1,2 l} \widetilde{N}_{1,2 l}} \times \\
{\left[R_{1,2 l}^{(1)}\left(-i c, i \xi_{<}\right) R_{1,2 l}^{(3)}\left(-i c, i \xi_{>}\right)+a_{l}^{(m)} R_{1,2 l}^{(3)}(-i c, i \xi)\right]} \\
\left.H_{1 \xi}\right|_{\eta=0}=-\frac{4 i k^{2}}{c \xi \sqrt{\xi_{0}^{2}+1}} \sum_{l=1}^{\infty} \frac{(-1)^{l} b_{l}^{(m)} P_{2 l}^{1^{\prime}}(0)}{\widetilde{\rho}_{1,2 l} \widetilde{N}_{1,2 l}} \times \\
\left.H_{1 \eta}\right|_{\xi=\xi_{1}}=\frac{\left[R_{1,2 l}^{(1)}(-i c, i \xi)+c_{l}^{(m)} R_{1,2 l}^{(3)}(-i c, i \xi)\right]}{c^{2} \sqrt{\left(\xi_{0}^{2}+1\right)\left(\xi_{1}^{2}+1\right)\left(\xi_{1}^{2}+\eta^{2}\right)} \times} \\
\sum_{l=1}^{\infty} \frac{(-1)^{l} b_{l}^{(m)}}{\widetilde{\rho}_{1,2 l} \widetilde{N}_{1,2 l}} \frac{S_{1,2 l}(-i c, \eta)}{R_{1,2 l}^{(3)}\left(-i c, i \xi_{1}\right)} .
\end{gathered}
$$

The diffracted far field is:

$$
\left.E_{\varphi}^{d}\right|_{c \xi \rightarrow \infty} \sim \frac{e^{i k r}}{k r} \frac{4 k^{2} Z}{\sqrt{\left(\xi_{0}^{2}+1\right)}} \sum_{l=1}^{\infty} \frac{a_{l}^{(m)}}{\widetilde{\rho}_{1,2 l} \widetilde{N}_{1,2 l}} S_{1,2 l}(-i c, \cos \theta) .
$$

In the limit when the cavity recedes to infinity:

$$
\left.E_{\varphi}^{d}\right|_{c \xi_{1} \rightarrow \infty} \sim-\left.E_{1 \varphi}\right|_{c \xi_{1} \rightarrow \infty} \sim \frac{4 k^{2} Z}{\sqrt{\left(\xi_{0}^{2}+1\right)}} \frac{1}{1+\zeta} \Sigma^{(m)},
$$

where

$$
\begin{aligned}
\Sigma^{(m)}= & \sum_{l=1}^{\infty} \frac{(-1)^{l}}{\widetilde{\rho}_{1,2 l} \widetilde{N}_{1,2 l}} \frac{R_{1,2 l}^{(1)^{\prime}}(-i c, i 0)}{R_{1,2 l}^{(3)^{\prime}}(-i c, i 0)} \times \\
& R_{1,2 l}^{(3)}\left(-i c, i \xi_{0}\right) R_{1,2 l}^{(3)}(-i c, i \xi) S_{1,2 l}(-i c, \eta),
\end{aligned}
$$

where, obviously, different ranges of $\eta$ apply for $z \leq 0$ and $z \geq 0$.

\section{Dipole SOURCES INSIDE THE CAVITY}

\section{A. Electric dipole}

Consider an electric dipole located on the $\mathrm{z}$ axis at $(\xi=$ $\left.\xi_{0}, \eta=-1\right)$ inside the cavity and axially oriented. The field of such a dipole in the presence of an infinite PEC plane at $z=0$, i.e. when the cavity recedes to infinity $\left(\xi_{1} \rightarrow \infty\right)$ and there is no coupling hole, leads to the configuration of Fig. 2 with dipole and image dipole interchanged. Therefore, the sum 
of incident and reflected fields leads to (11) with $Y$ replaced by $Y_{1}$ :

$$
\begin{array}{r}
H_{1 \varphi}^{i}+H_{1 \varphi}^{r}=-\frac{4 i k^{2} Y_{1}}{\sqrt{\xi_{0}^{2}+1}} \sum_{l=0}^{\infty} \frac{(-1)^{l}}{\widetilde{\rho}_{1,2 l+1} \widetilde{N}_{1,2 l+1}} \times \\
R_{1,2 l+1}^{(1)}\left(-i c, i \xi_{<}\right) R_{1,2 l+1}^{(3)}\left(-i c, i \xi_{>}\right) S_{1,2 l+1}(-i c, \eta) .
\end{array}
$$

The total magnetic field is:

$$
\begin{aligned}
H_{\varphi} & =H_{1 \varphi}^{i}+H_{1 \varphi}^{r}+H_{1 \varphi}^{s}, & & \text { for } z \leq 0, \\
& =H_{\varphi}^{d}, & & \text { for } z \geq 0,
\end{aligned}
$$

where $H_{1 \varphi}^{s}$ is the perturbation introduced in the field inside the cavity by the presence of the cavity and the coupling hole, whereas $H_{\varphi}^{d}$ is the field that enters the half-space $z \geq 0$ through the hole $\xi=0$. These fields are given by:

$$
\begin{aligned}
& H_{1 \varphi}^{s}=-\frac{4 i k^{2} Y_{1}}{\sqrt{\xi_{0}^{2}+1}} \sum_{l=0}^{\infty} \frac{(-1)^{l} B_{l}^{(e)}}{\widetilde{\rho}_{1,2 l+1} \widetilde{N}_{1,2 l+1}}\left[R_{1,2 l+1}^{(1)}(-i c, i \xi)+\right. \\
&\left.+C_{l}^{(e)} R_{1,2 l+1}^{(3)}(-i c, i \xi)\right] S_{1,2 l+1}(-i c, \eta) \\
& H_{\varphi}^{d}=-\frac{4 i k^{2} Y}{\sqrt{\xi_{0}^{2}+1}} \sum_{l=0}^{\infty} \frac{(-1)^{l} A_{l}^{(e)}}{\widetilde{\rho}_{1,2 l+1} \widetilde{N}_{1,2 l+1}} R_{1,2 l+1}^{(3)}(-i c, i \xi) \times \\
& S_{1,2 l+1}(-i c, \eta)
\end{aligned}
$$

which is the same as (13) with $a_{l}^{(e)}$ replaced by $A_{l}^{(e)}$. By imposing the boundary conditions:

$$
\begin{aligned}
A_{l}(e) & =-B_{l}^{(e)} C_{l}^{(e)} \\
A_{l}^{(e)} & =\frac{\zeta}{\Delta^{(e)}} R_{1,2 l+1}^{(1)}(-i c, i 0)\left[R_{1,2 l+1}^{(1)}\left(-i c, i \xi_{0}\right)+\right. \\
& \left.c_{l}^{(e)} R_{1,2 l+1}^{(3)}\left(-i c, i \xi_{0}\right)\right] \\
B_{l}(e) & =\frac{-1}{\Delta^{(e)}}\left[\zeta R_{1,2 l+1}^{(1)}(-i c, i 0) R_{1,2 l+1}^{(3)}\left(-i c, i \xi_{0}\right)-\right. \\
& \left.(1+\zeta) R_{1,2 l+1}^{(3)}(-i c, i 0) R_{1,2 l+1}^{(1)}\left(-i c, i \xi_{0}\right)\right] \\
\Delta^{(e)} & =\zeta R_{1,2 l+1}^{(1)}(-i c, i 0)+(1+\zeta) c_{l}^{(e)} R_{1,2 l+1}^{(3)}(-i c, i 0)
\end{aligned}
$$

with $c_{l}^{(e)}$ given by (18). In particular, the far field $\left.H_{\varphi}^{d}\right|_{c \xi \rightarrow \infty}$ is given by (24) with $a_{l}^{(e)}$ replaced by $A_{l}^{(e)}$.

\section{B. Magnetic dipole}

The total electric field produced by an axially oriented magnetic dipole located at $\left(\xi=\xi_{0}, \eta=-1\right)$ inside the cavity is:

$$
\begin{aligned}
E_{\varphi} & =E_{1 \varphi}^{i}+E_{1 \varphi}^{r}+E_{1 \varphi}^{s}, & & \text { for } z \leq 0 \\
& =E_{\varphi}^{d}, & & \text { for } z \geq 0
\end{aligned}
$$

The field $E_{1 \varphi}^{i}+E_{1 \varphi}^{r}$ due to the dipole in the presence of an infinite metal plane (cavity receded to infinity, $c \xi_{1} \rightarrow \infty$, and no hole) is obtained at once from (36) by replacing $Z$ with $Z_{1}$ and introducing a -1 factor to account for reversal $\left(180^{\circ}\right.$ phase shift) in the dipole orientation when imaged:

$$
\begin{array}{r}
E_{1 \varphi}^{i}+E_{1 \varphi}^{r}=\frac{4 k^{2} Z_{1}}{\sqrt{\xi_{0}^{2}+1}} \sum_{l=1}^{\infty} \frac{(-1)^{l}}{\widetilde{\rho}_{1,2 l} \widetilde{N}_{1,2 l}} R_{1,2 l}^{(1)}\left(-i c, i \xi_{<}\right) \times \\
R_{1,2 l}^{(3)}\left(-i c, i \xi_{>}\right) S_{1,2 l}(-i c, \eta) .
\end{array}
$$

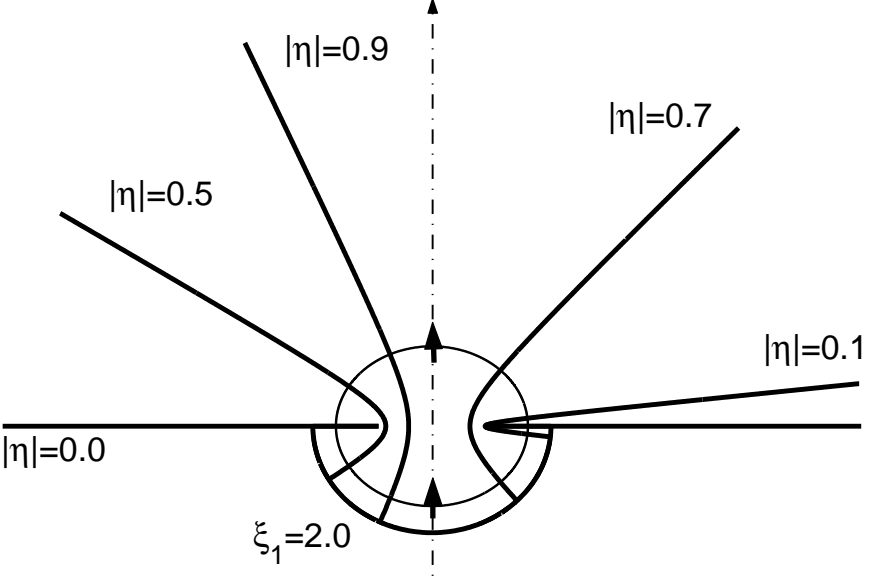

Fig. 3. Geometry for the computation of field quantities. The figure shows the locations of the dipole sources along the coordinate line $\xi=1.5$. However, only one source is considered for each case.

The perturbation due to the cavity and coupling hole is

$$
\begin{array}{r}
E_{1 \varphi}^{s}=\frac{4 k^{2} Z_{1}}{\sqrt{\xi_{0}^{2}+1}} \sum_{l=1}^{\infty} \frac{(-1)^{l} B_{l}^{(m)}}{\widetilde{\rho}_{1,2 l} \widetilde{N}_{1,2 l}}\left[R_{1,2 l}^{(1)}(-i c, i \xi)+\right. \\
\left.C_{l}^{(m)} R_{1,2 l}^{(3)}(-i c, i \xi)\right] S_{1,2 l}(-i c, \eta) .
\end{array}
$$

whereas the field radiated into the half-space $\geq 0$ through the coupling hole is:

$$
E_{\varphi}^{d}=\frac{4 k^{2} Z}{\sqrt{\xi_{0}^{2}+1}} \sum_{l=1}^{\infty} \frac{(-1)^{l} A_{l}^{(m)}}{\widetilde{\rho}_{1,2 l} \widetilde{N}_{1,2 l}} R_{1,2 l}^{(3)}(-i c, i \xi) S_{1,2 l}(-i c, \eta)
$$

which is (38) with $a_{l}^{(m)}$ replaced by $-A_{l}^{(m)}$; the same replacement yields the far field from (45). Imposition of the boundary conditions yields:

$$
\begin{aligned}
& B_{l}^{(m)} C_{l}^{(m)}=-\zeta A_{l}^{(m)} \\
& A_{l}^{(m)}=\frac{R_{1,2 l}^{(1)^{\prime}}(-i c, i 0)}{\Delta^{(m)}}\left[R_{1,2 l}^{(1)}\left(-i c, i \xi_{0}\right) R_{1,2 l}^{(3)}\left(-i c, i \xi_{1}\right)-\right. \\
& \left.R_{1,2 l}^{(3)}\left(-i c, i \xi_{0}\right) R_{1,2 l}^{(1)}\left(-i c, i \xi_{1}\right)\right] \\
& B_{l}^{(m)}=\frac{-R_{1,2 l}^{(3)}\left(-i c, i \xi_{1}\right)}{\Delta^{(m)}}\left[\zeta R_{1,2 l}^{(1)^{\prime}}(-i c, i 0) R_{1,2 l}^{(3)}\left(-i c, i \xi_{0}\right)-\right. \\
& \left.(1+\zeta) R_{1,2 l}^{(3)^{\prime}}(-i c, i 0) R_{1,2 l}^{(1)}\left(-i c, i \xi_{0}\right)\right]
\end{aligned}
$$

where

$$
\begin{aligned}
\Delta^{(m)}= & \zeta R_{1,2 l}^{(1)^{\prime}}(-i c, i 0) R_{1,2 l}^{(3)}\left(-i c, i \xi_{1}\right)- \\
& (1+\zeta) R_{1,2 l}^{(3)^{\prime}}(-i c, i 0) R_{1,2 l}^{(1)}\left(-i c, i \xi_{1}\right)
\end{aligned}
$$

\section{NUMERICAL RESULTS}

Computations of the oblate spheroidal functions were performed using some of the Fortran subroutines reported in [7], which were compared with those provided in [8]. The latter contains Fortran and Mathematica(c)subroutines. Another source for spheroidal functions that contains Mathematica(c)subroutines is [9]. Power series expansions for 
spheroidal wave functions with small argument $c$ are discussed in [10]. The computations of our series are similar to those encountered in [11] and [12]. In particular, in order to achieve convergence, use of the technique described in [13] was made.

As examples of the computation of some field expressions derived in this paper, we consider the evaluation of the quantities $H_{\varphi}$ and $E_{\varphi}$, since they act as scalar potentials according to (5-7) and (31-33).

One application of interest is the evaluation of the field that penetrates the cavity when the dipole source is located outside the cavity or, conversely, the field that is diffracted out of the cavity when the dipole source is located inside the cavity. We chose to evaluate the field along the coordinate lines $|\eta|=$ constant, see Figs. 1 and 3.

All the diagrams shown in Figs. 4-6 show the field quantities as a function of the dimensionless variable $2 z /(d|\eta|)$ since this quantity corresponds to

$$
\left\{\begin{aligned}
\xi, \text { when } z & \geq 0 \\
-\xi, \text { when } z & \leq 0
\end{aligned}\right.
$$

In fact, $\eta$ changes sign across the plane $z=0$ and $\xi \geq 0$, so that $2 z /(d|\eta|)$ may be looked upon as $|\xi|$ that is associated with a positive sign outside the cavity and with a negative sign inside the cavity. Different values of the dimensionless parameter $c=k d / 2$ are chosen. A physical meaning for $c$ is found by observing that $d / \lambda=c / \pi$ and, in particular, that $d=\lambda$ when $c=\pi$.

The results shown in Figs. 4-5 correspond to the cavity of Fig. 3 with $\xi_{1}=2$ for the dipole source located at $\left(\xi_{0}=1.5, \eta= \pm 1\right)$. These two figures show results for three different values of the ratio $\zeta=Z / Z_{1}: 1 / 2,1,2$. As an example of an electric dipole source located outside the cavity, Fig. 4 shows the results for the total magnetic field $\left|H_{\varphi}\right|$ computed using (12) and evaluated along the coordinate

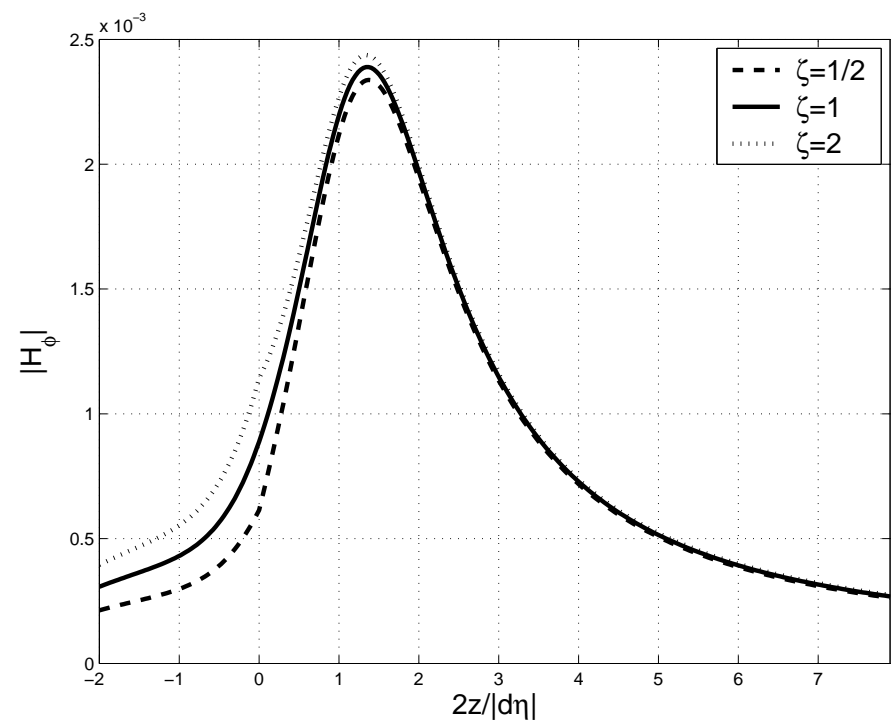

Fig. 4. Total magnetic field $\left|H_{\varphi}\right|$ due to an electric dipole located outside the cavity at $\left(\xi_{0}=1.5, \eta_{0}=1\right)$ and evaluated along the coordinate line $|\eta|=0.7$ of Fig. 3 for $c=1$. Results shown correspond to: $\zeta=1$ (solid line), $\zeta=0.5$ (dashed line), and $\zeta=2$ (dotted line).

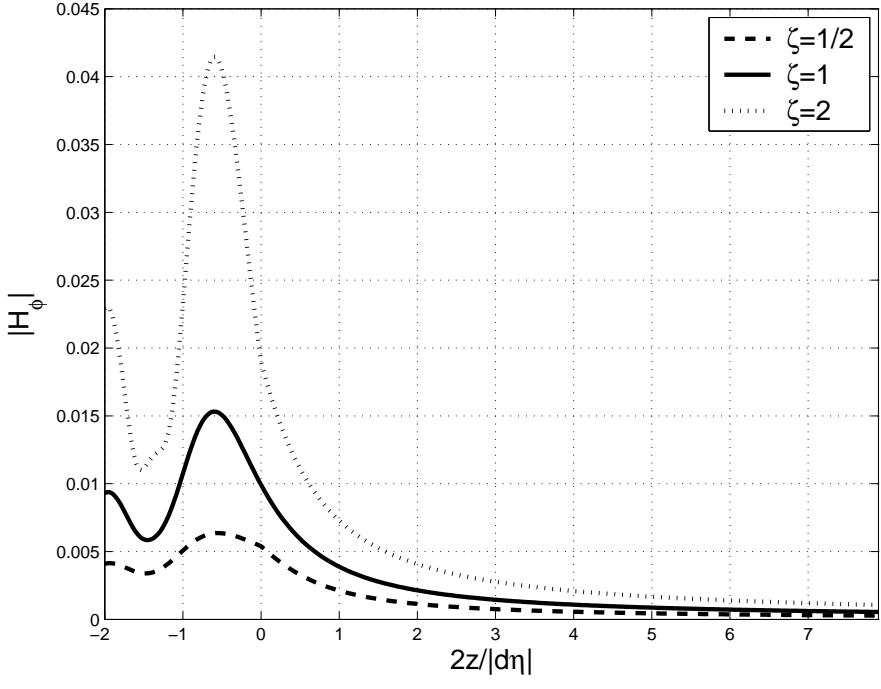

Fig. 5. Total magnetic field $\left|H_{\varphi}\right|$ due to an electric dipole located inside the cavity at $\left(\xi_{0}=1.5, \eta_{0}=-1\right)$ and evaluated along the coordinate line $|\eta|=0.1$ of Fig. 3 for $c=6$. Results shown correspond to: $\zeta=1$ (solid line), $\zeta=0.5$ (dashed line), and $\zeta=2$ (dotted line).

line $|\eta|=0.7$ of Fig. 3. Another result is given in Fig. 5, that shows the total magnetic field $\left|H_{\varphi}\right|$, computed using (49), for an electric dipole source located inside the cavity and evaluated along the coordinate line $|\eta|=0.1$ of Fig. 3. These two results appear to show that the fields are rather insensitive to the variation of the material properties only when the source is located outside the cavity.

The next numerical result is provided to show the spatial variation of the field. Hence, in Fig. 6 the total electric field $\left|E_{\varphi}\right|$ is computed using (56) with $\zeta=1$ and $c=3$ along three different curves: $|\eta|=0.1,0.7,0.9$.

Finally, Fig. 7 shows a contour plot of the magnitude of the electric field due to a magnetic source outside the cavity. The

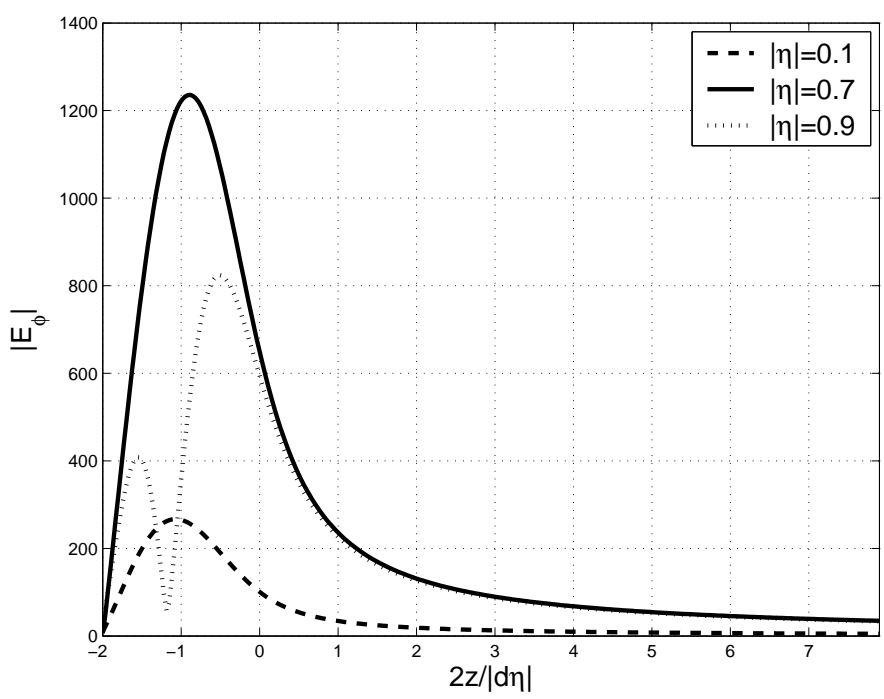

Fig. 6. Total electric field $\left|E_{\varphi}\right|$ due to a magnetic dipole located inside the cavity at $\left(\xi_{0}=1.5, \eta_{0}=-1\right)$ and evaluated for $\zeta=1$ and $c=3$. The results are computed along three lines $|\eta|=$ const and correspond to $|\eta|=0.1$ (solid-line), $|\eta|=0.7$ (dashed-line) and $|\eta|=0.9$ (dotted-line). 


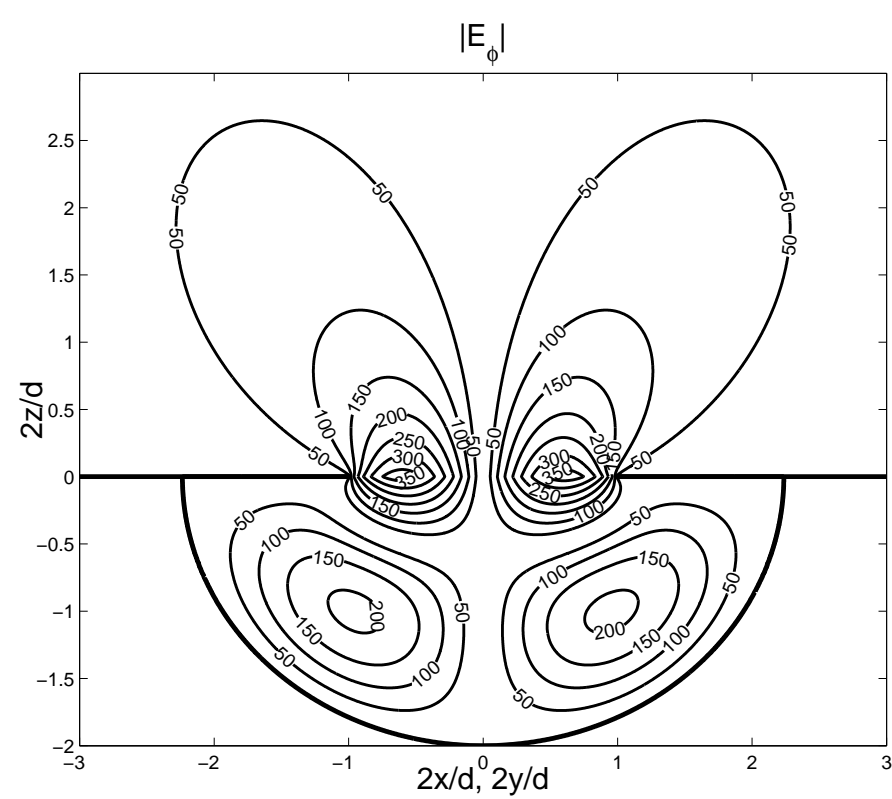

Fig. 7. Contour plot of the electric field $\left|E_{\varphi}\right|$ due to a magnetic dipole located outside the cavity at $\left(\xi_{0}=1.5, \eta_{0}=1\right)$ and evaluated for $\zeta=1$ and $c=3$. This plot shows the total electric field inside the cavity computed using (38), while outside the cavity only the scattered field (37) is considered.

contour plot was obtained by computing the total field, given by (38), inside the cavity and only the scattered field, given by (37), outside the cavity. In fact, the incident and reflected field contribution, given by (36), is the same that would be obtained from a perfectly conducting plane and, in addition, is much stronger than the scattered field close to the source. Therefore, the incident and reflected field contribution was removed to better appreciate the effect of the aperture and the cavity.

As a final remark, the quantities that correspond to the sum of incident and reflected fields given in (11), (36), (48), (57) were evaluated using the direct expression for the field radiated by a dipole source and its image, instead of the series expansions using oblate spheroidal functions.

The computation time of the results reported in figures 46 is less than 2 seconds for the evaluation of the field along coordinate lines, for which 300 points were considered. The computations of the contour plot required about 1 hour and 40 minutes to evaluate the field in a rectangular grid of 1'000'000 points. All simulations were run on a personal computer at $3.06 \mathrm{GHz}$.

\section{CONCLUSiOnS}

Analytical results for a new canonical problem involving a spheroidal cavity, sharp edges, two isorefractive media and a dipole sources located along the axis of symmetry and axially oriented were presented. This new analytical solution enriches the catalog of electromagnetic problems for which exact solutions are known. This solution is important, for example, to conduct a mutual validation with other methods, such as discussed in [11]-[12]. Numerical results were provided and discussed.
The reciprocity theorem allows us to calculate the axial component of the field on the $\mathrm{z}$ axis produced by an arbitrarily located and oriented dipole, from the solutions derived in the previous sections.

Future research will compare the results obtained in this paper with numerical solutions of integral equations. Also, the formulas obtained herein will be specialized to the lowfrequency case $c<<1$, thereby providing a test case for more general theories that describe coupling to a cavity via a small aperture.

\section{ACKNOWLEDGEMENTS}

The authors are thankful to the Reviewers for their comments that helped improve the quality of this paper.

\section{APPENDIX}

\section{SOME PROPERTIES OF OBLATE SPHEROIDAL FUNCTIONS}

The notation is that of Flammer [2]. We limit our considerations to the functions of interest in this paper: the angular functions $S_{1, n}(-i c, \eta)$; the radial functions of the first kind $R_{1, n}^{(1)}(-i c, i \xi)$; and the radial functions of the third kind $R_{1, n}^{(3)}(-i c, i \xi)$. The subscripts $n$ and $l$ are integers.

\section{1) Wronskian relation:}

$$
\begin{gathered}
R_{1, n}^{(1)}(-i c, i \xi) R_{1, n}^{(3)^{\prime}}(-i c, i \xi)-R_{1, n}^{(1)^{\prime}}(-i c, i \xi) R_{1, n}^{(3)}(-i c, i \xi)= \\
\frac{i}{c\left(\xi^{2}+1\right)}
\end{gathered}
$$

where the prime means the derivative with respect to $\xi$.

2) Special values:

$$
\begin{aligned}
S_{1,2 l}(-i c,-\eta) & =-S_{1,2 l}(-i c, \eta) \\
S_{1,2 l+1}(-i c,-\eta) & =S_{1,2 l+1}(-i c, \eta) \\
S_{1,2 l}(-i c, 0) & =0 \\
S_{1,2 l+1}(-i c, 0) & =P_{2 l+1}^{1}(0)=\frac{(-1)^{l}(2 l+2) !}{2^{2 l+1} l !(l+1) !} \\
\left.\frac{\partial}{\partial \eta} S_{1,2 l}(-i c, \eta)\right|_{\eta=0} & =\left.\frac{\partial}{\partial \eta} P_{2 l}^{1}(\eta)\right|_{\eta=0}=P_{2 l}^{1^{\prime}}(0)= \\
& \frac{(-1)^{(l-1)}(2 l+2) !}{2^{2 l}(l-1) !(l+1) !} \\
\left.\frac{\partial}{\partial \eta} S_{1,2 l+1}(-i c, \eta)\right|_{\eta=0} & =S_{1,2 l+1}^{\prime}(-i c, 0)=0
\end{aligned}
$$

where Ferrer's definition of associated Legendre functions is used.

$$
\begin{aligned}
R_{1,2 l}^{(1)}(-i c, 0) & =0 \\
\left.\frac{\partial}{\partial \xi} R_{1,2 l+1}^{(1)}(-i c, i \xi)\right|_{\xi=0} & =R_{1,2 l+1}^{(1)^{\prime}}(-i c, i 0)=0
\end{aligned}
$$


3) Asymptotic expansions:

$$
\begin{aligned}
\left.R_{1,2 l}^{(1)}(-i c, i \xi)\right|_{c \xi \rightarrow \infty} & \sim(-1)^{l} \frac{\sin c \xi}{c \xi} \underset{\operatorname{Im} c>0}{\longrightarrow} \\
& i(-1)^{l} \frac{e^{-i c \xi}}{2 c \xi} \\
\left.R_{1,2 l+1}^{(1)}(-i c, i \xi)\right|_{c \xi \rightarrow \infty} & \sim(-1)^{l+1} \frac{\cos c \xi}{c \xi} \underset{\operatorname{Im} c>0}{\longrightarrow} \\
\left.R_{1,2 l}^{(3)}(-i c, i \xi)\right|_{c \xi \rightarrow \infty} & \sim-i(-1)^{l+1} \frac{e^{-i c \xi}}{2 c \xi} \frac{e^{i c \xi}}{c \xi} \\
\left.R_{1,2 l+1}^{(3)}(-i c, i \xi)\right|_{c \xi \rightarrow \infty} & \sim(-1)^{l+1} \frac{e^{i c \xi}}{c \xi}
\end{aligned}
$$

4) Limit $d=0$ : When $d \rightarrow 0$ (hence $c \rightarrow 0$ ), the oblate spheroidal coordinate system reduces to the spherical coordinate system $(r, \theta, \varphi)$.

$$
\begin{aligned}
\lim _{d \rightarrow 0} S_{1, n}(-i c, \eta) & =P_{n}^{1}(\cos \theta) \\
\lim _{d \rightarrow 0} R_{1, n}^{(1)}(-i c, i \xi) & =\sqrt{\frac{\pi}{2 k r}} J_{n+\frac{1}{2}}(k r) \\
\lim _{d \rightarrow 0} R_{1, n}^{(3)}(-i c, i \xi) & =\sqrt{\frac{\pi}{2 k r}} H_{n+\frac{1}{2}}^{(1)}(k r)
\end{aligned}
$$

\section{REFERENCES}

[1] J.J. Bowman, T.B.A. Senior, and P.L.E. Uslenghi, Electromagnetic and Acoustic Scattering by Simple Shapes, Hemisphere Publishing Corporation, New York, 1987.

[2] C. Flammer, Spheroidal wave functions, Stanford University Press, 1957.

[3] P.L.E. Uslenghi, "Exact radiation from two axisymmetric structures containing isorefractive materials," in Proc. IEEE Antennas and Propagat. Intl. Symp., 1999, vol. 4, p. 2880.

[4] N. G. Alexopoulos, G.A. Tadler, and P.L.E. Uslenghi, "Scattering from spheroidal composite objects," J. Franklin Inst., vol. 309, pp. 147-162, 1980.

[5] A. R. Sebak and B.P. Sinha, "Scattering by a conducting spheroidal object with dielectric coating at axial incidence," IEEE Trans. Antennas Propagat., vol. 40, no. 3, pp. 268-274, March 1992.

[6] P.L.E. Uslenghi, "Exact penetration, radiation and scattering for a slotted semielliptical channel filled with isorefractive material," IEEE Trans. Antennas Propagat., 2003, to appear in the June 2004 issue.

[7] S. Zhang and J. M. Jin, Computation of Special Functions, Wiley, New York, 1996

[8] W. J. Thompson, Atlas for computing mathematical functions, Wiley, New York, 1997.

[9] L-W. Li, X-K. Kang, and M-S. Leong, Spheroidal wave functions in electromagnetic theory, Wiley, New York, 2001.

[10] G. C. Kokkorakis and J. A. Roumeliotis, "Power series expansions for spheroidal wave functions with small arguments," J. Comput. Appl. Math., vol. 139, no. 1, pp. 95-127, Feb 2002.

[11] D. Erricolo, M.D. Lockard, C.M. Butler, and P.L.E. Uslenghi, "Numerical analysis of penetration, radiation and scattering for a slotted semielliptical channel filled with isorefractive material," IEEE Trans. Antennas Propagat., 2003, submitted.

[12] D. Erricolo, M.D. Lockard, C.M. Butler, and P.L.E. Uslenghi, "Comparison among currents on surfaces inside and near a semielliptical channel filled with isorefractive material that backs a slotted plane: currents computed by analytical formulas and by integral equation methods," in Proc. Intl. Conf. on Electromagnetics in Advanced Applications (ICEAA'03), Torino, Italy, Sept 2003.

[13] D. Erricolo, "Acceleration of the convergence of series containing Mathieu functions using Shanks transformation," IEEE Antennas and Wireless Propagation Letters, vol. 2, pp. 58-61, 2003.

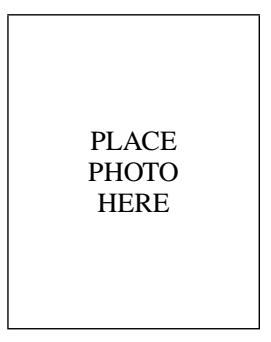

Cristian Berardi (S'02) received a Bachelor of Science in Electrical Engineering from the Politecnico di Milano, Milan, Italy in 2001, and the Master of Science in Electrical and Computer Engineering from the University of Illinois at Chicago in 2003. Currently, he is pursuing the Laurea degree in Electrical Engineering from the Politecnico di Milano. His research interests are in electromagnetic scattering and power electronics

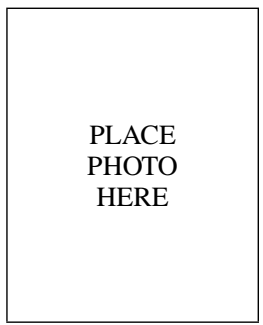

Danilo Erricolo (S'97 - M'99 - SM'03) received the Laurea degree of Doctor in Electronics Engineering (summa cum laude) from the Politecnico di Milano, Italy, in 1993, and the Ph.D. degree in Electrical Engineering and Computer Science from the University of Illinois at Chicago in 1998. He is a research scientist and a lecturer in the College of Engineering of the University of Illinois at Chicago where he also is the Associate Director of the Electromagnetics Laboratory. He is the Senior Associate Editor for the IEEE Antennas and Wireless Propagation Letters. His research interests are in computational electromagnetics, wireless communications, electromagnetic scattering, and electromagnetic compatibility. $\mathrm{He}$ has authored or co-authored more than 50 publications in international journals and conferences. Dr. Erricolo was awarded twice both the Andrew Foundation Fellowship and the Beltrami Foundation Fellowship. In 2002 he was elected full member of the United States National Committee of the International Union of Radio Science (USNC-URSI) Commission B.

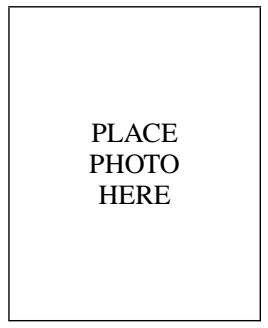

Piergiorgio L. E. Uslenghi (SM '70 - F '90) For biography and photo, see this Transactions, June 2004 